\title{
Funktionelle Dyspepsie: Besserung im Lauf der Zeit
}

\author{
Bis zu $40 \%$ der Bevölkerung in den \\ westlichen Industrieländern leiden \\ mehr oder weniger ausgeprägt an \\ einer funktionellen Dyspepsie. Wenig \\ ist darüber bekannt, wie sich die \\ Symptomatik dieser Patienten lang- \\ fristig entwickelt.
}

— Eine Kohorte von 476 Patienten der Universitätsklinik Löwen, bei denen zwischen 1998 und 2002 eine funktionelle Dyspepsie diagnostiziert worden war, wurde telefonisch kontaktiert und befragt, wie sich ihre Symptomatik entwickelt hatte.

Bei 17,4\% waren die Beschwerden verschwunden, bei $38,3 \%$ verbessert, bei $30,8 \%$ unverändert und bei $13,4 \%$ verschlechtert. Es bestand nur eine sehr schwache Korrelation zwischen der Dyspepsiesymptomatik beim ersten Besuch und dem Ausmaß der Dyspepsie beim Follow-up.
Als wichtigste Parameter für eine lang anhaltende und sich kaum bessernde klinische Symptomatik erwiesen sich der Dyspepsiescore bei der Eingangsuntersuchung und die dispositionnelle Ängstlichkeit. Weiterhin stellte man einen gewissen Trend der Assoziation mit einem Gewichtsverlust, depressiven Verstimmungen, einer chronischen Müdigkeit und mit Symptomen des Colon irritabile fest.

\section{Kommentar}

Der Gastroenterologe, dem diese Patienten zur oberen Endoskopie überwiesen werden, findet meist nichts und ist der Meinung, dass er für diese Patienten nichts tun könne. Der überweisende Hausarzt setzt aber oft zu hohe Erwartungen an das Ergebnis der fachärztlichen Untersuchung. Letztlich fühlt sich der Patient mit seinem Leiden allein gelassen. Doch zeigt die Studie, dass bei etwa der Hälfte der Patienten mit einer Besserung der Beschwerden im Lauf einer längeren Zeit zu rechnen ist. Die klare Assoziation zwischen einem Persistieren der Symptomatik und einer ängstlichen Persönlichkeitsstruktur sowie einer psychiatrischen Komorbidität lässt den Gedanken aufkommen, dass diese Patienten zwar aufgrund ihrer Symptomatik den Gastroenterologen beschäftigen, bei ihm aber wahrscheinlich gar nicht optimal betreut sind.

H. S. FÜESSL =

\section{- S. Kindt et al.}

Longitudinal and cross-sectional factors associated with long-term clinical course in functional dyspepsia: a 5-year follow-up study. Am. J. Gastroenterol. 106 (2011) 340-348

\section{Meditation lindert starke Schmerzen}

\begin{abstract}
Meditation kann die Konzentration und Aufmerksamkeit erhöhen. Für die Medizin besonders interessant ist die Hemmwirkung auf Schmerzen und das bereits nach vier Sitzungen.
\end{abstract}

- Forscher der Wake Forst University in Winston-Salem (USA) haben den schmerzlindernden Effekt der Meditation nicht nur quantitativ gemessen, sondern auch die Vorgänge im Gehirn verfolgt. 15 Versuchspersonen wurden in das Meditieren eingewiesen. Nach kurzer Übung setzte die Wirkung ein. Das Training dauerte insgesamt knapp eineinhalb Stunden. Gewählt wurde eine Art der Meditation, bei der sich die Meditierenden vor allem auf das Atmen konzentrieren.

Um den Zusammenhang zwischen Schmerzempfinden und Meditation zu prüfen, wurde bei den Probanden ein Wärme- schmerz $\left(50{ }^{\circ} \mathrm{C}\right)$ am Bein ausgelöst. Die Versuchsteilnehmer protokollierten ihr Schmerzempfinden. Die meisten beschrieben den Schmerz als stark oder intensiv. Mittels funktioneller Kernspintomografie wurde die Schmerzreaktion im Gehirn aufgezeichnet. Nach der ersten Testreihe wurden die Probanden in die Meditation eingewiesen. Dann wurde erneut getestet.

Nach der Meditation wurden die Schmerzen zu $40 \%$ weniger intensiv und zu 57\% weniger unangenehm empfunden. Das sind Werte, die mit manchen Schmerzmedikamenten nicht erreicht werden.

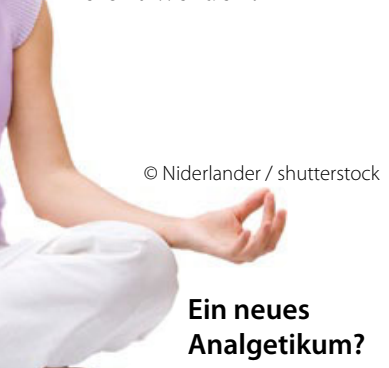

\section{Kommentar}

Dass Meditation die Schmerzen lindert, ist bereits mehrfach belegt worden. Doch die Hirnaktivitäten wurden unter diesen Bedingungen bisher nicht gemessen. Die Meditation reduziert die Aktivität im Gyrus postcentralis, einer Region, die für Ort und Intensität der Schmerzempfindung wichtig ist. Auch in anderen Hirnarealen sind Reaktionen gefunden worden wie z. B. im orbitofrontalen Cortex, der die Sinneseindrücke bewertet. Die Autoren meinen, dass die Meditation gerade wegen dieser Wirkungsvielfalt so effektiv ist. Jedoch fehlt eine echte Kontrollgruppe. Es ist deshalb nicht geklärt, ob die Schmerzlinderung allein durch die bloße Erwartungshaltung bedingt ist.

K. MALBERG

- F. Zeidan et al.

Brain mechanisms supporting the modulation of pain by mindfulness meditation. J. Neurosci. 31 (2011) 5540-5548 